\title{
Review Article \\ Circulating microRNAs: A Potential Role in Diagnosis and Prognosis of Acute Myocardial Infarction
}

\author{
Ali Sheikh Md Sayed, ${ }^{1}$ Ke Xia, ${ }^{1,2}$ Tian-Lun Yang, ${ }^{1}$ and Jun Peng ${ }^{3}$ \\ ${ }^{1}$ Cardiology Department, Xiangya Hospital, Central South University, No. 87 Xiangya Road, Changsha 410078, China \\ ${ }^{2}$ Cardiovascular Division, Department of Medicine, Center for Vascular Biology and Inflammation, Brigham and Women's Hospital, \\ Harvard Medical School, Boston, MA 02108, USA \\ ${ }^{3}$ Department of Pharmacology, School of Pharmaceutical Sciences, Central South University, Changsha 410078, China
}

Correspondence should be addressed to Tian-Lun Yang; tianluny@163.com and Jun Peng; junpeng@csu.edu.cn

Received 14 August 2013; Accepted 30 September 2013

Academic Editor: Marco Peluso

Copyright (C) 2013 Ali Sheikh Md Sayed et al. This is an open access article distributed under the Creative Commons Attribution License, which permits unrestricted use, distribution, and reproduction in any medium, provided the original work is properly cited.

\begin{abstract}
Rapid and correct diagnosis of acute myocardial infarction (AMI) plays a crucial role in saving patients' life. Although some biomarkers (such as cardiac troponin and creatine kinase) are available for AMI diagnosis so far, there is still a clinical need for novel biomarkers, which can reliably rule in or rule out AMI immediately on admission. Circulating microRNAs (miRNAs) are a potential choice for novel biomarkers in AMI diagnosis and prognosis with high sensitivity and specificity. Circulating microRNAs are endogenous miRNAs that are detectable in whole blood, serum, or plasma in a highly stable form. Until now, around 20 circulating miRNAs were reported to be closely associated with AMI. In this minireview, we summarized recent available data on the correlation between circulating miRNAs and AMI. Some miRNAs, such as miR-208, miR-499, miR-133, and miR-1, were given special attention, since they may have a potential prospect in diagnosis and prognosis of AMI.
\end{abstract}

\section{Introduction}

Coronary artery disease (CAD) is a very common health problem in the developed as well as developing countries. Acute myocardial infarction (AMI) is one of the leading causes for death worldwide, and it is the single largest cause of death in the United States, responsible for 1 out of every 6 deaths $[1,2]$. Rapid and correct diagnosis of AMI plays an important role in therapy and prognosis for this disease. Over the past two decades, huge progress has been made in the diagnosis, treatment, and prognosis of AMI. Particularly, the progress in biomarkers for AMI attracted a great deal of attention. Currently, cardiac troponins are the most common biomarkers used for diagnosis of AMI in clinical practice. However, there is still a clinical need for novel biomarker, which is able to reliably rule in or rule out AMI immediately on admission. MicroRNAs (miRNAs) seem to be a promising candidate of novel biomarker for early diagnosis of AMI [3]. In the last few years, the dysregulation of tissue expression levels of miRNAs has been directly linked to cardiac disease.
In addition to expression changes in tissues, more recent studies have indicated that miRNAs are detectable in serum, plasma, urine, and other body fluids in a highly stable form that is protected from endogenous RNase activity [4]. Altered circulating miRNA concentrations have been detected in patients with AMI [5], acute coronary syndrome (ACS) [6], stable coronary artery disease [7], heart failure [8], coronary atherosclerosis, cardiac arrhythmia, cardiomyopathy, and cardiac hypertrophy [9]. This review will focus on a potential role of circulating miRNAs in diagnosis of AMI as novel biomarkers.

\section{Circulating miRNAs}

miRNAs are endogenous, small ( 22 nucleotides), singlestranded, noncoding RNAs that regulate gene expression at the posttranscriptional level by binding to the $3^{\prime}$ untranslated regions (UTRs) of their target mRNAs [10]. When miRNAs completely bind to their target mRNAs, degradation of 
the target mRNAs will be initiated. But, when the miRNAs bind partially, the translation of mRNA is repressed. miRNAs are involved in multiple biological processes, such as proliferation, migration, differentiation, secretion, excitation, conduction, cell cycle, ageing, and apoptosis, by changing protein expression of potential candidate targets [11].

miRNAs have recently been detected in serum or plasma, which are referred to circulating miRNAs. Despite intense research, the origin of circulating miRNAs remains largely unknown. A number of studies have reported that miRNAs are actively secreted in microvesicles or exosomes from different cell types [12], which are a likely source of circulating miRNAs. So far, around 121 miRNAs were identified in exosomes from mast cells [13], and sphingomyelinase 2 (nSMase2), a rate-limiting enzyme for ceramide biosynthesis, controls the secretion of exosomes to the extracellular matrix [14]. In addition to microvesicles or exosomes, microparticles and lipoprotein complexes (such as high-density lipoprotein complexes) are other possible sources of circulating miRNAs.

Microparticles are larger than exosomes of plasma membrane origin, contain miRNAs, and actively control cell communication process [15]. High-density lipoprotein (HDL) has been reported to transport endogenous miRNAs and deliver them to recipient cells with functional targeting capabilities besides its basic role as a delivery vehicle for excess cellular cholesterol. Interestingly, the human HDL-miRNA profile from normal subjects is significantly different than familial hypercholesterolemia subjects. The most abundant miRNAs associated with HDL from normal subjects were hsa-miR135a, hsa-miR-188-5p, and hsa-miR-877, whereas the most abundant familial hypercholesterolemia HDL-miRNAs were hsa-miR-223, hsa-miR-105, and hsa-miR-106a. Therefore, human HDL not only transports endogenous miRNAs, but also can display different functions under different conditions and act as potential mediators in gene regulation and intercellular communication. The detection of circulating miRNAs in the plasma and serum indicates that miRNAs may fulfill biological functions outside the cell and act as potential biomarkers for diseases [16]. The human genome is estimated to encode up to 1000 miRNAs and over 100 miRNAs in serum were identified from healthy subjects [17].

\section{Stability and Isolation of Circulating microRNAs}

Human miRNAs isolated from plasma are highly stable in boiling water, in solution with very high or low $\mathrm{pH}$. Plasma miRNA levels remain stable when it is subjected to prolonged room temperature incubation or freeze-thawed multiple times. It has been reported that endogenous plasma miRNAs exist in a form that is resistant to plasma RNase activity [18]. Compared to the endogenous plasma miRNAs, rapid degradation was observed within minutes when synthetic miRNAs (corresponding to caenorhabditis elegans miRNAs cel-miR-39, cel-miR-54, and cel-miR-238) were added into human plasma. Inactivating the RNase activity before the addition, the exogenous miRNAs escaped degradation. Therefore, exogenous plasma miRNAs are not stable and are susceptible to rapid degradation, whereas native circulating miRNAs are stable and resistant to RNase activity [19]. There are multiple factors that account for the protection of circulating miRNAs against RNase-dependent degradation. For example, circulating miRNAs are not free in blood, which are normally included in microvesicles or exosomes. Furthermore, the circulating miRNAs can form the protein-miRNA complexes with some special proteins (such as nucleolar RNA-binding protein, nucleophosmin 1) to resist the degradation [20].

Although circulating microRNAs are stable, reproducible isolation of cell-free miRNAs with high purity is a technical challenge for multiple reasons. First, plasma or serum contains very low amounts of RNA. The yield of RNA from small volume of plasma or serum (usually less than $1 \mathrm{~mL}$ ) falls below the limit of accurate quality control by regular methods. Second, high concentration of proteins in plasma or serum could potentially interfere with the sample preparation and the detection assay. Thus, avoiding protein contamination and hemolysis (lysis of red blood cells) in serum/plasma samples are important challenges to overcome. Finally, some of the larger small RNA species frequently used as reference genes (such as U6 RNA, cel-miR-39) to normalize the data of $\mathrm{qPCR}$ are present in extremely low concentrations in serum or plasma. Therefore, great care must be taken when choosing controls (miR-156 is an option) for normalization. Fortunately, with circulating microRNAs well recognized for potential diagnostic biomarkers in a wide range of diseases and biological processes, more commercial kits for high quality of circulating microRNAs isolation are available in the market.

\section{Circulating miRNAs of Cardiac Origin and Acute Myocardial Infarction}

Rapid and correct diagnosis is crucial to treatment and prognosis of AMI. Up to date, cardiac troponins and creatine kinase-MB are the most commonly used biomarkers for AMI, but their clinical value is limited in many cases. Secreted by cardiac cells and accumulated in blood, miRNAs are expected to reflect cardiac injury in response to cardiovascular risk factors and various pathological conditions. Thus, the circulating cardiac-specific or -enriched miRNAs (such as miR208, miR-499, and miR-133) may provide unique biomarkers for diagnostic and therapeutic interventions of AMI [21].

4.1. $m i R-208$. miR-208 is a cardiac-enriched microRNA and found at much higher levels during cardiac tissue injury. It is also involved in stress, hormone, or electric stimulation in cardiac cells $[22,23]$. During cardiac development, miR-208 exerts a regulatory role in the production of the myosin heavy chain [24]. It has been reported that plasma concentration of circulating miR-208 levels was significantly increased following isoproterenol-induced rat model of myocardial injury. But this phenomenon was not observed after renal injury, indicating that circulating miR-208 is specific for cardiac injury. There was a good correlation between the changes 
in plasma miR-208 and cardiac troponin I (cTnI), a classical biomarker of AMI [25].

4.1.1. $m i R-208 a$. Through microarray analysis, miR-208a was absolutely identified to be expressed in the human heart. miR-208a was undetectable in plasma of healthy individuals or non-AMI patients (such as patients with acute kidney injury, chronic renal failure, stroke, or trauma), but it was easily detected in 90.9\% AMI patients and in 100\% AMI patients within $4 \mathrm{~h}$ of the onset of chest pain while cTnI was not yet affected, suggesting that miRNAs may leak into the bloodstream at an earlier stage of myocardial injury (the biological peak of troponins is $\sim 18 \mathrm{~h}$ after AMI). It is reasonable to speculate that circulating miR-208a has advantages over cTnI (or $\mathrm{cTnT}$ ) in distinguishing pure AMI from patients with renal disease, stroke, and trauma [5].

Interestingly, the elevation of circulating miR-208a in AMI could be persistent in adult mouse but not in human. A possible explanation for this phenomenon might be due to the different miRNA expression pattern between human and mice. miR-208a is encoded within an intron of $\alpha$-cardiac muscle myosin heavy chain gene (Myh6), while miR-208b, a close family member of miR-208, is encoded within an intron of $\beta$-cardiac muscle myosin heavy chain gene ( $M y h 7)$. There exists high degree of homology between miR-208a and miR$208 \mathrm{~b}$ in mice but not in human. It is likely that the persistently elevated plasma level of miR-208a after AMI might be the result of miR-208b interference [23].

4.1.2. miR-208b. In case of acute ST segment elevation myocardial infarction (STEMI) patients, the levels of plasma miR-208b increased 3,000-fold compared to healthy controls within $12 \mathrm{~h}$ after infarction. Peak values of miR-208b were well correlated with peak troponin I (cTnI) and the ejection fraction, indicating a possible role for circulating miR-208b as a biomarker in diagnosis of STEMI or in prediction of long-term complications [26]. In AMI patients, plasma miR-208b was increased 1600-fold compared with healthy subjects. The receiver operating characteristic (ROC) curve analysis revealed an area under the ROC curve (AUC) of 0.94 , suggesting that miR-208b was an ideal biomarker for AMI. The changes in plasma level of miR-208b were consistent with plasma troponin- $\mathrm{T}$, indicating that miR-208b was released from injured cardiomyocytes like troponin-T [27]. However, it is not all of the studies that supported the role of miR-208 as a biomarker for AMI. Adachi et al. reported that circulating miR-208b level appeared to be very low in AMI patients, and it was a useful plasma biomarker for AMI diagnosis [28]. Another study has recently demonstrated that circulating miR-208b might be used as a biomarker for AMI but it did not show any advantages over cTNT for AMI diagnosis [29].

4.2. $m i R-499$. The gene encoded miR-499 is embedded within a ventricular-specific myosin heavy chain gene, which is almost exclusively expressed in the heart [30]. Plasma level of miR-499 was obviously increased in all patients with AMI but it was below the limit of detection for acute coronary syndromes, congestive heart failure, and for all healthy controls.
These results indicated that circulating miR- 499 might be a useful biomarker for the diagnosis of AMI [28]. It has been demonstrated that plasma level of miR-499 increased 250fold or 100-fold in patients with acute STEMI or AMI compared with healthy controls [26]. ROC curve analysis revealed an area under the curve of 0.92 , which is correlated with circulating $c \operatorname{TnT}$. In AMI patients with chest pain $<3 \mathrm{~h}$, miR499 was positive in $93 \%$ of patients while the positive rate for hs-cTnT was $88 \%$, suggesting that miR-499 is a sensitive and novel biomarker for the diagnosis of AMI [31]. However, results of ROC curve analysis from Li et al.'s study showed no advantage for plasma miR-499 over cTNT in AMI diagnosis.

Circulating miR-499-5p, a family member of miR-499, was increased more than 80 -fold in geriatric patients with acute non-ST elevation myocardial infarction (NSTEMI) compared with healthy subjects. The ROC curve analysis showed that diagnostic accuracy of miR-499-5p was higher than that of TNT (AUC of miR-499-5p versus cTnT versus hs-cTnT: 0.86 versus 0.68 versus 0.70 ). Thus, circulating miR$499-5 p$ is a potential biomarker and its accuracy is superior to conventional $\mathrm{cTnT}$ for the diagnosis of acute NSTEMI in the elderly patients [32]. Recently, a study demonstrated that miR-499-5p was higher in MI patients and was negatively correlated with the value of left ventricular ejection fraction, strongly suggesting that circulating miR-499-5p might be used for the evaluation of mortality risk [33].

4.3. miR-133. In a rat AMI model, plasma level of miR133a was increased at $1-3 \mathrm{~h}$, peaked at 3-12 $\mathrm{h}$, and decreased at $12-24 \mathrm{~h}$ after coronary artery ligation. In AMI patients, miR-133 plasma level was substantially higher compared with healthy controls. A positive correlation was also reported between the elevated plasma miR-133 and cTNI. These results provided evidence that miR-133 is a powerful biomarker for the diagnosis of AMI [5]. However, there were some different reports on circulating miR-133. In a report, no significant difference in plasma level of miR-133 had been found between AMI patients and healthy subjects [34]. Circulating miR133 also showed no significant changes in AMI patients with tachyarrhythmia or bradyarrhythmia compared to those in patients without arrhythmias. Plasma levels of miR-133a and miR-133b, family members of miR-133, were reported to be upregulated in acute STEMI patients compared with healthy subjects, which was peaked at about 156 minutes after occurrence of infarction, consistent with time course of change in cTNI level. In contrast, the level of circulating miR133a was not significantly changed in mice subjected to acute hind-limb ischemia [35].

4.4. miR-1. The plasma level of miR-1 was reported to markedly increased in rats with AMI or in AMI patients, which was only mildly increased in patients with non-AMI coronary heart disease or patients with other cardiovascular diseases [5]. In a rat model of AMI induced by coronary ligation, serum miR-1 level was rapidly increased around 200fold after AMI with a peak at $6 \mathrm{~h}$, and the miR-1 level returned to basal levels at 3 days after AMI, which displayed a strong positive correlation with myocardial infarct size. In a clinical 
TABLE 1: Summary of circulating miRNAs and AMI.

\begin{tabular}{|c|c|c|c|c|c|}
\hline miRNAs & Diseases & Species & Upregulation/downregulation & Correlation with cardiac troponin & Reference \\
\hline miR-208a & AMI & Human & Up & Positive & {$[5]$} \\
\hline miR-208a & AMI & Rat & Up & & {$[23]$} \\
\hline \multirow{3}{*}{$\operatorname{miR}-208 b$} & Acute STEMI & Human & Up & Positive & {$[26]$} \\
\hline & AMI & Human & Up & & {$[27]$} \\
\hline & AMI & Human & Up & Positive & {$[29]$} \\
\hline \multirow{5}{*}{ miR-499 } & Acute STEMI & Human & Up & Positive & {$[26]$} \\
\hline & AMI & Human & Up & Positive & {$[31]$} \\
\hline & NSTEMI & Human & Up & Positive & {$[32]$} \\
\hline & AMI & Human & Up & & {$[29]$} \\
\hline & AMI & Human & Up & & {$[33]$} \\
\hline \multirow{3}{*}{ miR-133 } & AMI & Rat & Up & & {$[5]$} \\
\hline & AMI & Human & UP & & {$[35]$} \\
\hline & AMI & Human & Up & Positive & {$[38]$} \\
\hline \multirow{5}{*}{ miR-1 } & AMI & Rat & Up & & {$[5]$} \\
\hline & AMI & Human, rat & Up & & {$[36]$} \\
\hline & AMI & Human & Up & Positive & {$[34]$} \\
\hline & AMI & Human & Up & & {$[26]$} \\
\hline & AMI & Human & Up & & {$[29]$} \\
\hline $\operatorname{miR}-328$ & AMI & Human & Up & Positive & {$[43]$} \\
\hline miR-1291 & AMI & Human & Up & & {$[36]$} \\
\hline miR-21 & AMI & Human & Up & & {$[44]$} \\
\hline miR-30, miR-195 & AMI & Human & Up & & {$[45]$} \\
\hline miR-let-7b & AMI & Human & Down & & {$[45]$} \\
\hline miR-378 & AMI & Rat & Down & & {$[46]$} \\
\hline miR-126 & AMI & Human & Down & & {$[40]$} \\
\hline
\end{tabular}

AMI: acute myocardial infarction; STEMI: ST segment elevation myocardial infarction; NSTEMI: non-ST segment elevation myocardial infarction.

study, the level of circulating miR-1 was increased nearly 100fold in AMI patients compared with healthy subjects and showed a positive correlation with serum creatine kinaseMB (CK-MB) level. In another separate clinical study, plasma miR-1 level was significantly higher in AMI patients compared with non-AMI subjects and the level was returned to normal on discharge following medication. Interestingly, the increased plasma miR-1 was not associated with age, gender, blood pressure, diabetes mellitus, or other established biomarkers for AMI including cTnI and CK-MB. Moreover, in a necrosis model of cultured cardiac cells, miR-1 was found to be released into the culture medium and was stable at least for $24 \mathrm{~h}$. Collectively, these results strongly support that circulating miR-1 might be a novel sensitive biomarker for AMI diagnosis [36].

\section{Circulating miRNAs of Noncardiac Origin and Acute Myocardial Infarction}

In theory, the change of circulating miRNAs after AMI is due to the injured heart, which releases miRNAs into the blood. However, there is evidence to show that many changes in circulating miRNAs may not originate from the injured heart. In a recent study, whole-genome miRNA expression in peripheral total blood samples from AMI patients was analyzed and 121 miRNAs were reported to be dysregulated in comparison to healthy controls. Among them, miR-30c and miR-145 levels were found to be correlated with infarct size, miR-1291 and miR-663b were highly sensitive and specific for the discrimination of cases from controls, and a unique signature of 20 miRNAs was shown to predict AMI with even higher power (specificity $96 \%$, sensitivity $90 \%$, and accuracy 93\%) [37]. These results indicate that miRNA signatures derived from peripheral blood cells could be also used as novel, sensitive biomarkers for the diagnosis of AMI.

miR-126 is specifically and highly expressed in human endothelial cells. It has a potential role in angiogenesis and in regulation of endothelial homeostasis and vascular integrity $[38,39]$. Circulating plasma miR-126 was significantly downregulated in AMI patients compared with healthy subjects. The plasma concentration of miR-126 also showed a good correlation with the plasma concentration of cTnI in AMI [40]. Circulating levels of miR-126 were decreased during transcoronary passage in patients with evidence of myocardial injury compared with noncoronary artery disease [41]. Recently, a prospective study has reported that circulating miR-126 has a positive association with fatal myocardial infarction [42].

So far, more and more circulating miRNAs, cardiac origin or noncardiac origin, were reported to be potential biomarkers for AMI. Table 1 is a summary of the correlation between 
circulating miRNAs and AMI from the recent literatures. As displayed in Table 1, at least 13 miRNAs were reported as potential biomarkers for AMI. Among them, cardiac-specific or -enriched miRNAs (such as miR-208, miR-499, miR-133, and miR-1) attracted more attention [25, 27, 28, 34]. Since miR-208 and miR-499 were only detected in heart while miR133 and miR-1 were highly expressed in both skeletal and cardiac muscle, in theory, miR-208 and miR-499 should have the advantages (such as more specific or sensitive) over miR133 and miR-1 in AMI diagnosis. Actually, in Wang et al.s study, they examined the plasma levels of miR-208, miR499, miR-133, and miR-1 in AMI patients and rat model of AMI. After careful comparison and analysis of the results, they concluded that among these four miRNAs, miR-208 is a more reliable biomarker for AMI diagnosis [5]. However, in Devaux et al.s study, they provided evidence that circulating miR-499 appeared clearly superior to circulating miR-208 for detecting cardiac damage [31]. Thus, it needs further studies to determine which one (miR-208 or miR-499) is the most potential biomarker for AMI diagnosis.

\section{Conclusion}

In summary, circulating miRNAs are resistant to endogenous ribonuclease activity and can be present in human plasma or serum in a remarkably stable form. It is believed that circulating miRNAs provide a novel class of minimal invasive biomarkers for AMI diagnosis. Until now, around 20 miRNAs were reported as potential biomarkers for AMI. However, most of the findings were based on relatively small samples, which lead to many divergences between different reports. Therefore, it is necessary to conduct independent and large cohort studies to identify those circulating miRNAs with real value in AMI diagnosis. So far, most of the studies focus on the role of circulating miRNAs as biomarkers for AMI diagnosis. The information regarding the prognostic impact of circulating miRNAs in AMI is still largely lacking. Actually, as ideal biomarkers, it is also important to be useful in AMI prognosis. Recently, we have found that plasma levels of miR-208b, miR-499, miR-149, and miR-424 were significantly elevated in AMI. Among them, miR-208b, miR-499, and miR-424 were rapidly decreased after percutaneous coronary intervention (PCI) (unpublished data), suggesting that miR208b, miR-499, and miR-424 might be potential candidates as biomarkers for AMI prognosis. It is a possible future direction to seek specific circulating miRNAs with potential value in AMI diagnosis as well as prognosis.

\section{Conflict of Interests}

All authors declare that there is no conflict of interests.

\section{Acknowledgments}

The authors would like to thank Dr. Umme Salma for revision of the paper. This work was supported by Specialized Research Fund for the Doctoral Program of Higher Education of China (no. 200805331178 to K. Xia), NuoMei
Foundation of China Branch, International Atherosclerosis Society (no. NMF 2008-003 to T. L. Yang), CGICC Foundation of Chinese Medical Association (no. 08010008 to K. Xia), Hunan Provincial Natural Science Foundation of China (no. 13JJ2008 to J. Peng), and Doctoral Fund of Ministry of Education of China (no. 20120162110056 to J. Peng).

\section{References}

[1] H. D. White and D. P. Chew, "Acute myocardial infarction," The Lancet, vol. 372, no. 9638, pp. 570-584, 2008.

[2] V. L. Roger, A. S. Go, D. M. Lloyd-Jones et al., "Heart disease and stroke statistics-2011 update: a report from the American heart association," Circulation, vol. 123, no. 4, pp. e18-e209, 2011.

[3] B. Lindahl, "Acute coronary syndrome-the present and future role of biomarkers," Clinical Chemistry and Laboratory Medicine, vol. 23, no. 3, pp. 1-8, 2013.

[4] S. S. C. Chim, T. K. F. Shing, E. C. W. Hung et al., "Detection and characterization of placental microRNAs in maternal plasma," Clinical Chemistry, vol. 54, no. 3, pp. 482-490, 2008.

[5] G. K. Wang, J. Q. Zhu, J. T. Zhang et al., "Circulating microRNA: a novel potential biomarker for early diagnosis of acute myocardial infarction in humans," European Heart Journal, vol. 31, no. 6, pp. 659-666, 2010.

[6] C. Widera, S. K. Gupta, J. M. Lorenzen et al., "Diagnostic and prognostic impact of six circulating microRNAs in acute coronary syndrome," Journal of Molecular and Cellular Cardiology, vol. 51, no. 5, pp. 872-875, 2011.

[7] S. Fichtlscherer, S. de Rosa, H. Fox et al., "Circulating microRNAs in patients with coronary artery disease," Circulation Research, vol. 107, no. 5, pp. 677-684, 2010.

[8] A. J. Tijsen, E. E. Creemers, P. D. Moerland et al., "MiR423-5p as a circulating biomarker for heart failure," Circulation Research, vol. 106, no. 6, pp. 1035-1039, 2010.

[9] Z. W. Pan, Y. J. Lu, and B. F. Yang, "MicroRNAs: a novel class of potential therapeutic targets for cardiovascular diseases," Acta Pharmacologica Sinica, vol. 31, no. 1, pp. 1-9, 2010.

[10] D. Baek, J. Villén, C. Shin, F. D. Camargo, S. P. Gygi, and D. P. Bartel, "The impact of microRNAs on protein output," Nature, vol. 455 , no. 7209, pp. 64-71, 2008.

[11] E. M. Small and E. N. Olson, "Pervasive roles of microRNAs in cardiovascular biology," Nature, vol. 469, no. 7330, pp. 336-342, 2011.

[12] J. Skog, T. Würdinger, S. van Rijn et al., "Glioblastoma microvesicles transport RNA and proteins that promote tumour growth and provide diagnostic biomarkers," Nature Cell Biology, vol. 10, no. 12, pp. 1470-1476, 2008.

[13] G. Camussi, M. C. Deregibus, S. Bruno, V. Cantaluppi, and L. Biancone, "Exosomes/microvesicles as a mechanism of cell-tocell communication," Kidney International, vol. 78, no. 9, pp. 838-848, 2010.

[14] N. Kosaka, H. Iguchi, Y. Yoshioka, F. Takeshita, Y. Matsuki, and T. Ochiya, "Secretory mechanisms and intercellular transfer of microRNAs in living cells," The Journal of Biological Chemistry, vol. 285, no. 23, pp. 17442-17452, 2010.

[15] M. P. Hunter, N. Ismail, X. Zhang et al., "Detection of microRNA expression in human peripheral blood microvesicles," PLoS ONE, vol. 3, no. 11, Article ID e3694, 2008.

[16] S. Gilad, E. Meiri, Y. Yogev et al., "Serum microRNAs are promising novel biomarkers," PLoS ONE, vol. 3, no. 9, Article ID e3148, 2008. 
[17] X. Chen, Y. Ba, L. Ma et al., "Characterization of microRNAs in serum: a novel class of biomarkers for diagnosis of cancer and other diseases," Cell Research, vol. 18, no. 10, pp. 997-1006, 2008.

[18] A. Turchinovich, L. Weiz, A. Langheinz, and B. Burwinkel, "Characterization of extracellular circulating microRNA," Nucleic Acids Research, vol. 39, no. 16, pp. 7223-7233, 2011.

[19] N. B. Y. Tsui, E. K. O. Ng, and Y. M. D. Lo, "Stability of endogenous and added RNA in blood specimens, serum, and plasma," Clinical Chemistry, vol. 48, no. 10, pp. 1647-1653, 2002.

[20] K. Wang, S. Zhang, J. Weber, D. Baxter, and D. J. Galas, "Export of microRNAs and microRNA-protective protein by mammalian cells," Nucleic Acids Research, vol. 38, no. 20, pp. 7248-7259, 2010.

[21] K. B. Margulies, "MicroRNAs as novel myocardial biomarkers," Clinical Chemistry, vol. 55, no. 11, pp. 1897-1899, 2009.

[22] E. van Rooij, L. B. Sutherland, X. Qi, J. A. Richardson, J. Hill, and E. N. Olson, "Control of stress-dependent cardiac growth and gene expression by a microRNA," Science, vol. 316, no. 5824, pp. 575-579, 2007.

[23] T. E. Callis, K. Pandya, H. Y. Seok et al., "MicroRNA-208a is a regulator of cardiac hypertrophy and conduction in mice," Journal of Clinical Investigation, vol. 119, no. 9, pp. 2772-2786, 2009.

[24] A. P. Malizia and D. Z. Wang, "MicroRNAs in cardiomyocyte development," Wiley Interdisciplinary Reviews: Systems Biology and Medicine, vol. 3, no. 2, pp. 183-190, 2011.

[25] X. Ji, R. Takahashi, Y. Hiura, G. Hirokawa, Y. Fukushima, and N. Iwai, "Plasma miR-208 as a biomarker of myocardial injury," Clinical Chemistry, vol. 55, no. 11, pp. 1944-1949, 2009.

[26] O. Gidlöf, P. Andersson, J. van der Pals, M. Götberg, and D. Erlinge, "Cardiospecific microRNA plasma levels correlate with troponin and cardiac function in patients with ST elevation myocardial infarction, are selectively dependent on renal elimination, and can be detected in urine samples," Cardiology, vol. 118, no. 4, pp. 217-226, 2011.

[27] M. F. Corsten, R. Dennert, S. Jochems et al., "Circulating MicroRNA-208b and MicroRNA-499 reflect myocardial damage in cardiovascular disease," Circulation: Cardiovascular Genetics, vol. 3, no. 6, pp. 499-506, 2010.

[28] T. Adachi, M. Nakanishi, Y. Otsuka et al., "Plasma microRNA 499 as a biomarker of acute myocardial infarction," Clinical Chemistry, vol. 56, no. 7, pp. 1183-1185, 2010.

[29] Y. Q. Li, M. F. Zhang, H. Y. Wen et al., "Comparing the diagnostic values of circulating microRNAs and cardiac troponin $\mathrm{T}$ in patients with acute myocardial infarction," Clinics, vol. 68 , no. 1 , pp. 75-80, 2013.

[30] J. T. C. Shieh, Y. Huang, J. Gilmore, and D. Srivastava, "Elevated miR-499 levels blunt the cardiac stress response," PLoS ONE, vol. 6, no. 5, Article ID e19481, 2011.

[31] Y. Devaux, M. Vausort, E. Goretti et al., "Use of circulating microRNAs to diagnose acute myocardial infarction," Clinical Chemistry, vol. 58, no. 3, pp. 559-567, 2012.

[32] F. Olivieri, R. Antonicelli, M. Lorenzi et al., "Diagnostic potential of circulating miR-499-5p in elderly patients with acute non ST-elevation myocardial infarction," International Journal of Cardiology, vol. 167, no. 2, pp. 531-536, 2013.

[33] O. Gidlöf, J. G. Smith, K. Miyazu et al., "Circulating cardioenriched microRNAs are associated with long-term prognosis following myocardial infarction," BMC Cardiovascular Disorders, vol. 13, article 12, 2013.
[34] J. Ai, R. Zhang, Y. Li et al., "Circulating microRNA-1 as a potential novel biomarker for acute myocardial infarction," Biochemical and Biophysical Research Communications, vol. 391, no. 1, pp. 73-77, 2010.

[35] Y. D’Alessandra, P. Devanna, F. Limana et al., "Circulating microRNAs are new and sensitive biomarkers of myocardial infarction," European Heart Journal, vol. 31, no. 22, pp. 27652773, 2010.

[36] Y. Cheng, N. Tan, J. Yang et al., "A translational study of circulating cell-free microRNA-1 in acute myocardial infarction," Clinical Science, vol. 119, no. 2, pp. 87-95, 2010.

[37] B. Meder, A. Keller, B. Vogel et al., "MicroRNA signatures in total peripheral blood as novel biomarkers for acute myocardial infarction," Basic Research in Cardiology, vol. 106, no. 1, pp. 1323, 2011.

[38] C. van Solingen, L. Seghers, R. Bijkerk et al., "Antagomirmediated silencing of endothelial cell specific microRNA-126 impairs ischemia-induced angiogenesis," Journal of Cellular and Molecular Medicine, vol. 13, no. 8, pp. 1577-1585, 2009.

[39] P. Mocharla, S. Briand, G. Giannotti et al., "AngiomiR-126 expression and secretion from circulating $\mathrm{CD} 34^{+}$and $\mathrm{CD} 14^{+}$ PBMCs: role for proangiogenic effects and alterations in type 2 diabetics," Blood, vol. 121, no. 1, pp. 226-236, 2013.

[40] G. Long, F. Wang, Q. Duan et al., "Human circulating microRNA-1 and microRNA-126 as potential novel indicators for acute myocardial infarction," International Journal of Biological Sciences, vol. 8, no. 6, pp. 811-818, 2012.

[41] S. de Rosa, S. Fichtlscherer, R. Lehmann, B. Assmus, S. Dimmeler, and A. M. Zeiher, "Transcoronary concentration gradients of circulating MicroRNAs," Circulation, vol. 124, no. 18, pp. 1936-1944, 2011.

[42] A. Zampetaki, P. Willeit, L. Tilling et al., "Prospective study on circulating MicroRNAs and risk of myocardial infarction," Journal of the American College of Cardiology, vol. 60, no. 4, pp. 290-299, 2012.

[43] R. Wang, N. Li, Y. Zhang, Y. Ran, and J. Pu, "Circulating micro RNAs are promising novel biomarkers of acute myocardial infarction," Internal Medicine, vol. 50, no. 17, pp. 1789-1795, 2011.

[44] M. R. Zile, S. M. Mehurg, J. E. Arroyo, R. E. Stroud, S. M. DeSantis, and F. G. Spinale, "Relationship between the temporal profile of plasma microRNA and left ventricular remodeling in patients after myocardial infarction," Circulation: Cardiovascular Genetics, vol. 4, no. 6, pp. 614-619, 2011.

[45] G. Long, F. Wang, Q. Duan et al., "Circulating miR-30a, miR-195 and let-7b associated with acute myocardial infarction," PLoS ONE, vol. 7, no. 12, Article ID e50926, 2012.

[46] J. Fang, X. W. Song, J. Tian et al., "Overexpression of microRNA378 attenuates ischemia-induced apoptosis by inhibiting caspase-3 expression in cardiac myocytes," Apoptosis, vol. 17, no. 4 , pp. 410-423, 2012. 


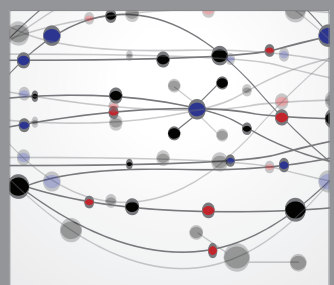

The Scientific World Journal
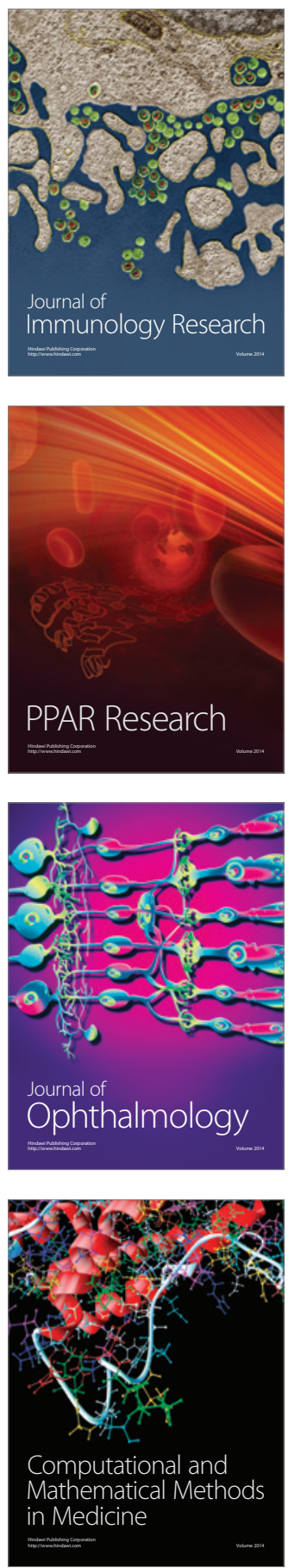

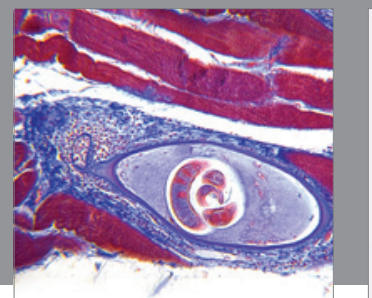

Gastroenterology

Research and Practice
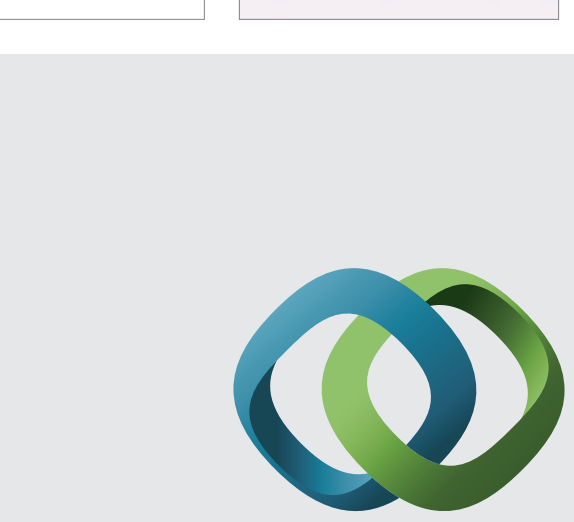

\section{Hindawi}

Submit your manuscripts at

http://www.hindawi.com
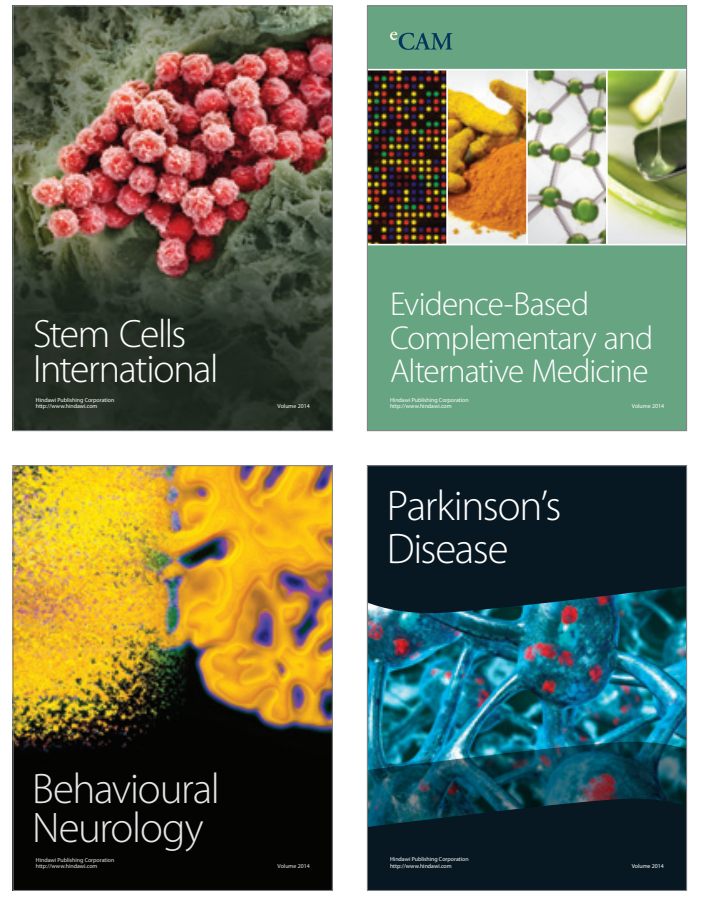
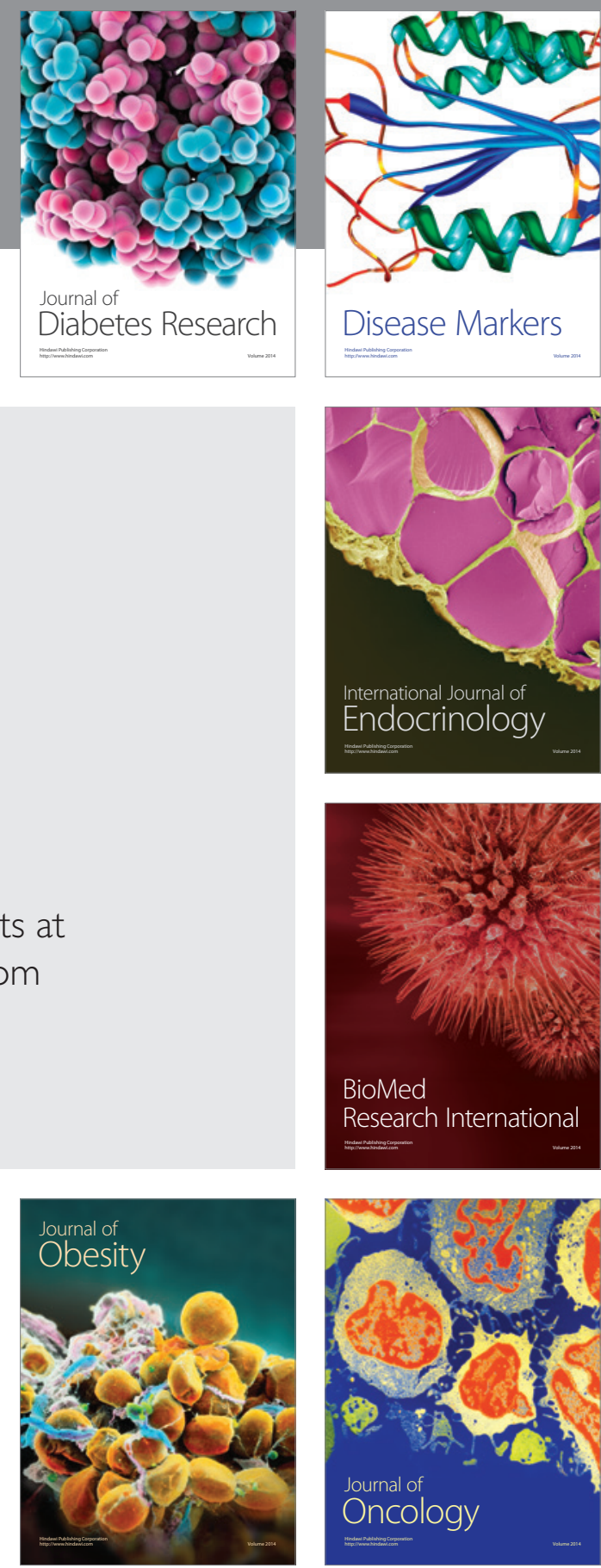

Disease Markers
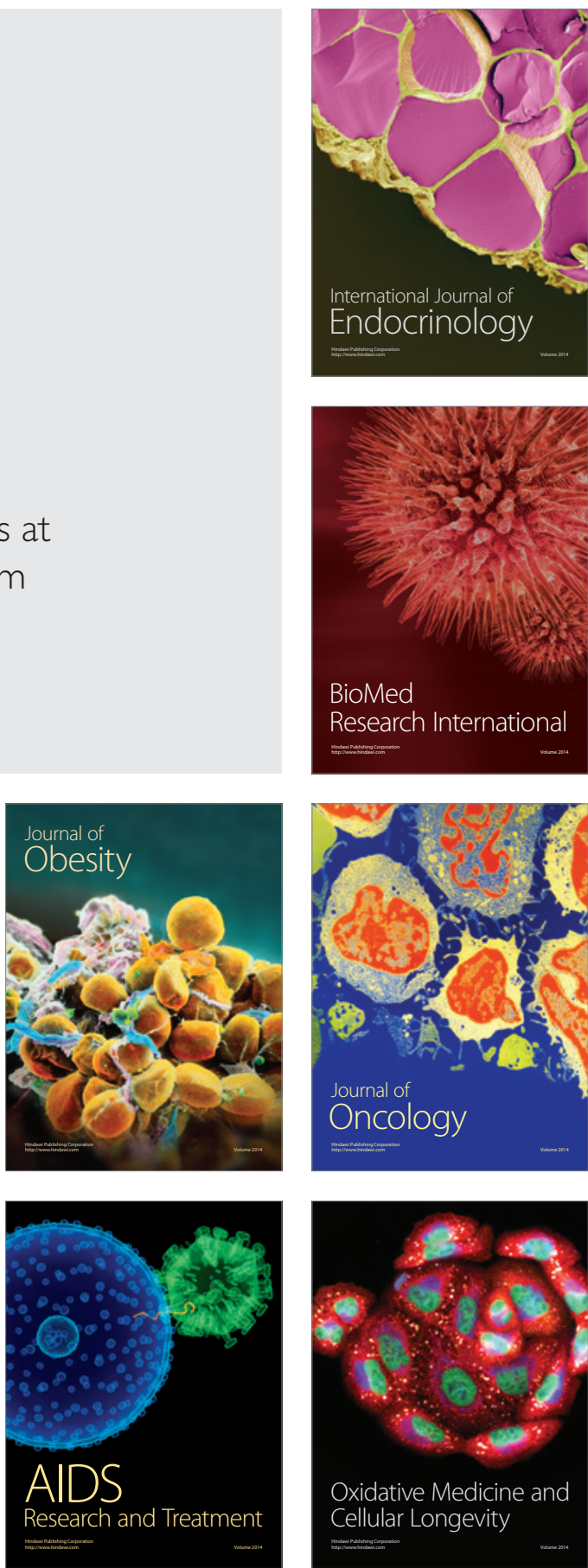